\title{
INHIBICIÓN DE Streptococcus mutans CON EXTRACTOS DE Rubus ulmifolius, Passiflora mollissima Y Vaccinium floribundum
}

\section{INHIBITION OF Streptococcus mutans WITH EXTRACTS OF Rubus ulmifolius, Passiflora mollissima AND Vaccinium floribundum}

\author{
Stalin Gustavo Santacruz Terán y Rudy Gissella Tagle González \\ Universidad Laica Eloy Alfaro de Manabí, vía a San Mateo s/n, Manta, Ecuador \\ Email: stalin.santacruz@gmail.com
}

\section{Información del artículo}

Tipo de artículo: Artículo original

Recibido:

05/08/2020

Aceptado:

27/11/2020

Licencia:

CC BY-NC-SA 4.0

Revista

ESPAMCIENCIA

11(2):75-79

DOI:

https://doi.org/10.51

260/revista_espamci

encia.v11i2.220

\section{Resumen}

Es conocido que el consumo excesivo de azúcares conlleva al desarrollo de caries dental y obesidad, entre otras enfermedades. Conociendo que algunos de los compuestos fenólicos tienen carácter antimicrobiano, en el presente estudio se evaluó la actividad antimicrobianan in vitro de mora (Rubus ulmifolius), taxo (Passiflora mollissima) y mortiño (Vaccinium floribundum) en concentraciones de 1,2,3\% frente a Streptococcus mutans, microorganismo responsable del desarrollo de caries dental, mediante el método de difusión con discos. El efecto de la adición de fruta a leche entera que proporciona el Programa de Alimentación Escolar se evaluó mediante análisis sensorial con la adición de taxo y mortiño al 1\%. Los resultados mostraron que extractos de mora, mortiño y taxo presentaron un halo de inhibición promedio a las $24 \mathrm{~h}$ de 1,34, 1,42, y 1,57 cm y a las 48 h $1,31,1,40$ y $1,46 \mathrm{~cm}$, respectivamente. Mortiño y taxo tuvieron igual actividad inhibitoria $(\mathrm{p}<0,05)$. Los extractos con concentraciones diferentes mostraron que la concentración de $3 \%$ presentó una mayor zona de inhibición con respecto a 1 y $2 \%$, siendo estas dos últimas estadísticamente iguales. Los resultados del análisis sensorial mostraron que la leche saborizada con mortiño tuvo mayor aceptación, con una calificación de "me gusta mucho" en una escala hedónica de 5 puntos, concluyendo que es la fruta recomendada para utilizar como saborizante en leche, sabiendo además que tiene alto poder inhibitorio frente al $S$. mutans.

Palabras clave: caries dental, actividad antimicrobiana, leche saborizada, poder inhibitorio

\begin{abstract}
It is known that excessive consumption of sugars leads to tooth decay and obesity, among other diseases. Some phenolic compounds have an antimicrobial character. In this study, the antimicrobial activity of blackberry (Rubus ulmifolius), taxa (Passiflora mollissima) and mortiño (Vaccinium floribundum) was evaluated in vitro in concentrations of 1,2,3\% against Streptococcus mutans, a microorganism responsible for the development of dental caries. The method of diffusion with discs was used. The effect of fruit addition to whole milk provided by the School Feeding Program was evaluated by sensory analysis with the addition of taxo and mortiño at $1 \%$. The results showed that blackberry, mortiño and taxo extracts presented an average inhibition halo at $24 \mathrm{~h}$ of $1.34,1.42$, and $1.57 \mathrm{~cm}$ and at $48 \mathrm{~h}$ of $1.31,1.40$ and $1.46 \mathrm{~cm}$, respectively. Mortiño and taxo had equal inhibitory activity $(\mathrm{p}<0.05)$. The extracts with different concentrations showed that the concentration of $3 \%$ presented a greater inhibition zone with respect to 1 and $2 \%$, being these last two statistically equal. The results of the sensory analysis showed that the mortiño-flavored milk had the greatest acceptance, with a classification of "I like it very much" in a 5-point hedonic scale. In conclusion, mortiño is the recommended fruit to use as milk flavoring, added to the fact that it also has high inhibitory power against $S$. mutans.
\end{abstract}

Keywords: dental caries, antimicrobial activity, flavored milk, inhibitory power 


\section{INTRODUCCIÓN}

La caries dental es uno de los padecimientos de salud más habituales en todo el mundo (GBDS, 2018). Según la OMS, la población más propensa a sufrir problemas son los niños de edad escolar, encontrándose que entre el $60 \%$ y el $90 \%$ de este grupo se ven afectados (PérezDomínguez et al., 2010).

La dieta desempeña un papel fundamental en el desarrollo de la caries dental. Muchos estudios epidemiológicos correlacionan el consumo de azúcar con la prevalencia de caries (Freire et al., 2016). Dentro del coctel de microorganismos responsables de la caries dental destaca el Streptococcus mutans (Arreguín-Cano et al., 2016).

Actualmente Ecuador a través de programas de alimentación escolar ofrece alimentos sólidos como galletas o granola y bebidas como leche saborizadas (PAE, 2017). En su información nutricional, la leche ofrecida por el programa de alimentación escolar (PAE) describe un contenido de $14 \mathrm{~g}$ de azúcar por cada $200 \mathrm{~g}$ de bebida. De acuerdo a este último valor no existe problema en el contenido de azúcar, sin embargo, hay que considerar el consumo de otros alimentos azucarados consumidos durante el día. El azúcar total consumido por un niño con el programa de alimentación escolar es de aproximadamente 24 gramos (barra de cereales 2,5 g, galletas $7 \mathrm{~g}$, leche saborizada $14 \mathrm{~g}$ ). La OMS recomienda una ingesta diaria de $50 \mathrm{~g}$ de azúcares en niños y $25 \mathrm{~g}$ si se desea obtener beneficios adicionales (Ruiz y Varela, 2017). Indudablemente, el azúcar total consumido por niños en edad escolar favorece el desarrollo de caries dentales (Duque de Estrada et al., 2006; Cid et al., 2008)

Además de su actividad antioxidante establecida, muchos compuestos fenólicos pueden exhibir una actividad antibacteriana significativa (Orhan et al., 2010; Lou et al., 2012). Dado que muchos extractos de plantas son ricos en compuestos fenólicos, esto es de particular interés para el desarrollo de alternativas naturales a los conservantes sintéticos en alimentos (Bouarab-Chibane et al., 2019), aplicaciones cosméticas (Kocevar y Lunder, 2018) y aplicaciones en medicina. Dentro de la odontología, Reyes et al. (2019a, 2019b) encontraron que los extractos de Prunus salicifoica y Vaccinium floribundum presentaron actividad inhibitoria contra $S$. mutans.

Los mecanismos de acción antibacteriana de los compuestos fenólicos aún no están completamente descifrados, pero se sabe que estos compuestos involucran muchos sitios de acción a nivel celular (Bouarab-Chibane et al., 2019). Varios autores explican esta actividad por la modificación de la permeabilidad de las membranas celulares, los cambios en diversas funciones intracelulares inducidos por la unión del hidrógeno de los compuestos fenólicos a las enzimas o por la modificación de la rigidez de la pared celular con pérdidas de integridad por diferentes interacciones con la membrana celular. (Cushnie y Lamb, 2011).

Vasco (2009), mostró que frutas cultivadas en el Ecuador poseen un alto contenido de compuestos fenólicos, tal es el caso de la mora (Rubus ulmifolius), taxo (Passiflora mollissima) o mortiño (Vaccinium floribundum), estas frutas están disponibles en el mercado ecuatoriano, pero debido a una falta de conocimiento actual su consumo es relativamente bajo, tanto como fruta así como en el desarrollo de productos de valor agregado, desaprovechando así sus posibles beneficios medicinales $\mathrm{y}$ funcionales.

Con base en la información previa, la presente investigación estudió el uso de extractos de mora, taxo y mortiño como inhibidores del desarrollo de Streptococcus mutans. Adicionalmente, se analizó la aceptabilidad de leches saborizadas preparadas con extractos de las tres frutas.

\section{MATERIALES Y MÉTODOS}

La leche empleada en el estudio fue leche entera UHT del Programa de Alimentación Escolar. La mora y taxo fueron adquiridas en el mercado de la ciudad de Manta, mientras que el mortiño se obtuvo de un mercado la ciudad de Quito.

Las frutas fueron sometidas a un proceso de selección para retirar las frutas que presentaron daños físicos, así como cualquier impureza. Las frutas seleccionadas se lavaron con agua potable, para posteriormente desintegrar $250 \mathrm{~g}$ de fruta con ayuda de una licuadora doméstica (Oster SW194DT, Reino Unido). La pulpa desintegrada se filtró en una tela gabardina lisa $6 \mathrm{oz}$ crudo (Telasxmetro, Argentina), eliminándose el material sólido. El material filtrado (extracto) se mezcló con leche entera para obtener porcentajes de extracto de 1, 2, 3\% en la leche final (leche saborizada).

\section{Análisis de inhibición in vitro de Streptococcus mutans}

Se utilizó el método de difusión en agar según la técnica estandarizada por el National Committee for Clinical Laboratory Standards (NCCLS, 2015). La evaluación de la actividad antibacteriana se realizó en cepas de Streptococcus mutans ATCC35668 (Minnesota, USA laboratorio Microbiologics). Para preparar el inóculo, las cepas liofilizadas fueron activadas en caldo BHI (Brain Heart Infusion Broth C5141 Criterion, USA), luego sembradas en cajas Petri con $20 \mathrm{~mL}$ de medio de cultivo agar sangre a $37^{\circ} \mathrm{C}$ durante $48 \mathrm{~h}$. Una vez activados los microorganismos en estudio, se preparó una suspensión bacteriana al 0,5 en la escala de Mc Farland (Santacruz and Castro, 2018), que representa una concentración de $1,5 \times 10^{8}$ UFC, misma que se inoculó en cajas Petri con agar sangre. Posteriormente, se colocó en las cajas Petri discos de papel filtro (Fisher Scientific Q2) de $5 \mathrm{~mm}$ de diámetro, que contenían $20 \mu \mathrm{L}$ de leche saborizada, previamente preparada en tres concentraciones más una 
muestra control (leche no saborizada). Las placas inoculadas se incubaron a una temperatura de $37^{\circ} \mathrm{C}$ durante 2 días, luego de lo cual se midió la zona de inhibición del crecimiento bacteriano. En todos los casos la evaluación se realizó por triplicado.

\section{Análisis de evaluación sensorial}

Con base en los resultados del análisis de inhibición, se preparó leche saborizada de las frutas a concentración del 1\%. Las leches saborizadas conjuntamente con la muestra control (leche no saborizada) se utilizaron en un análisis sensorial que permitió determinar la leche saborizada con mayor aceptación. Para ello se realizó una prueba de satisfacción con una escala hedónica de cinco puntos que fue de "me disgusta mucho" a "me gusta mucho", misma que permitió establecer el nivel de agrado de las muestras (Gaytán-Andrade et al., 2019). Para la prueba se contó con 40 panelistas no entrenados.

\section{Diseño experimental}

Se utilizó un diseño completamente al azar (DCA) con arreglo bifactorial $(3 \times 3+1)$, utilizando como control una leche entera no saborizada. Las variables independientes fueron el tipo de fruta, en 3 niveles, (mora, mortiño y taxo) y la concentración del extracto de fruta, en 3 niveles $(1,2$ y $3 \%$ ). La variable dependiente fue la zona de inhibición de $S$. mutans.

\section{Análisis estadístico}

El análisis de los resultados se realizó mediante análisis de varianza y prueba de medias de Tukey con un nivel de significancia del 5\% mediante el programa estadístico Infostat Versión 2015.

\section{RESULTADOS Y DISCUSIÓN}

\section{Análisis de inhibición in vitro de Streptococcus mutans}

El cuadro 1 muestra que la muestra control no presentó inhibición, mientras que la leche con $3 \%$ de extracto de fruta fue estadísticamente diferente y presentó mayor inhibición (diámetro de $1,58 \mathrm{~cm}$ ) que las muestras con otras concentraciones de fruta, luego de $24 \mathrm{~h}$ de estudio $(\mathrm{p}<0,05)$. El mismo comportamiento se observó a las $48 \mathrm{~h}$. No existió diferencia estadística entre las concentraciones de 1 y $2 \%$. Análisis de inhibición de $S$. mutans fueron realizados por Reyes et al. (2019a), quienes encontraron zonas de inhibición entre 0,83 y $1,73 \mathrm{~cm}$ por acción de extractos de mortiño y Reyes et al. (2019b) zonas de 0,88 $\mathrm{cm}$, por acción de extracto de mortiño deshidratado. Dichos valores son similares a los obtenidos en el presente trabajo.
Cuadro 1. Comparación de medias de la zona de inhibición de $S$. mutans luego de 24 y $48 \mathrm{~h}$ frente extractos de mora, mortiño y mora en concentraciones de 1,2 y $3 \%$

\begin{tabular}{ccccc}
\hline Concentración (\%) & \multicolumn{4}{l}{ Zona de inhibición } \\
\cline { 2 - 6 } & $\mathbf{2 4} \mathbf{~ h ( c m )}$ & $\mathbf{4 8} \mathbf{~ h}(\mathbf{c m})$ & \\
\hline $\mathbf{0}$ & $0,00^{\mathrm{A}}$ & $0,00^{\mathrm{A}}$ & & \\
$\mathbf{1}$ & $1,37^{\mathrm{B}}$ & $1,32^{\mathrm{B}}$ & & \\
$\mathbf{2}$ & $1,39^{\mathrm{B}}$ & $1,35^{\mathrm{B}}$ & & \\
$\mathbf{3}$ & $1,58^{\mathrm{C}}$ & $1,51^{\mathrm{C}}$ & & \\
\hline Medias con una letra & superíndice & común & no & son \\
significativamente diferentes $(\mathrm{p}<0,05)$ & & & \\
\end{tabular}

Los resultados de inhibición respecto al tipo de fruta mostraron que no existió diferencia en las zonas de inhibición luego de 24 y 48 h de incubación. El control mostró diferencia con todos los tratamientos $(\mathrm{p}<0,05)$. Las zonas de inhibición de mora y mortiño fueron iguales, con medias de 1,34 y $1,42 \mathrm{~cm}$, respectivamente, luego de $24 \mathrm{~h}$ de incubación (Cuadro 2).

Cuadro 2. Comparación de medias de la zona de inhibición del $S$. mutans luego de 24 y $48 \mathrm{~h}$ frente al tipo de fruta

\begin{tabular}{cccc}
\hline \multirow{2}{*}{ Fruta } & \multicolumn{3}{c}{ Zona de inhibición } \\
\cline { 2 - 4 } & $\mathbf{2 4} \mathbf{~ h} \mathbf{( c m})$ & $\mathbf{4 8} \mathbf{~ h} \mathbf{( c m )}$ \\
\hline Control & $0,00^{\mathrm{A}}$ & $0,00^{\mathrm{A}}$ & \\
Mora & $1,34^{\mathrm{B}}$ & $1,31^{\mathrm{B}}$ & \\
Mortiño & $1,42^{\mathrm{B}, \mathrm{C}}$ & $1,40^{\mathrm{B}, \mathrm{C}}$ & \\
Taxo & $1,57^{\mathrm{C}}$ & $1,46^{\mathrm{C}}$ & \\
\hline Medias con una letra superíndice & común no & son \\
significativamente diferentes $(\mathrm{p}<0,05)$ & & &
\end{tabular}

Los resultados mostraron mayores halos de inhibición para taxo y mortiño lo que indica una mayor actividad inhibitoria sobre la cepa de $S$. mutans por parte de estas dos frutas en relación a la mora. Vasco (2009) ubica al mortiño y al taxo como frutas con alto contenido de compuestos fenólicos. Posiblemente la presencia de flavonoides (Rodríguez et al., 2017) conjuntamente con la alta concentración de compuestos fenólicos podrían contribuir con el poder inhibitorio de S. mutans.

\section{Análisis de evaluación sensorial}

Los resultados de inhibición in vitro, mostraron que las frutas con mayor efecto inhibitorio frente a $S$. mutans fueron taxo y mortiño. La concentración de extracto escogida, con mayor poder inhibitorio sobre la bacteria, para saborizar la leche, fue del $1 \%$, esto con el objeto de no modificar la formulación original de la leche del PAE.

Los resultados de la prueba de comparación de medias mostraron que las tres muestras presentaron diferencias significativas entre sí (Cuadro 3). La muestra de mortiño presentó una media de 4,32, que corresponde a "me gusta moderadamente". La muestra control (no saborizada) presentó una media de 3,17 que equivale "me es 
indiferente" y la muestra saborizada con taxo obtuvo una media de 2,93 que correspondió a "me disgusta un poco". Con base en los resultados se pudo concluir que a pesar de que el taxo tuvo el mayor poder de inhibición frente a $S$. mutans, no se recomienda su uso como saborizante de leche por su bajo nivel de agrado.

Cuadro 3. Comparación de medias de evaluación sensorial de leche saborizada con extractos de taxo y mora en concentración de $1 \%$

\begin{tabular}{lccc}
\hline Bebida & Calificación & \\
\hline Leche + taxo & $2,93^{\mathrm{A}}$ & \\
Leche control & $4,17^{\mathrm{B}}$ & \\
Leche + mortiño & $4,32^{\mathrm{C}}$ & \\
\hline Medias con una letra superíndice común no & son \\
significativamente diferentes $(\mathrm{p}<0,05)$ & & &
\end{tabular}

\section{CONCLUSIONES}

Los análisis microbiológicos in vitro mostraron que la fruta que presentó mayor poder inhibitorio frente a $S$. mutans fue el taxo, seguida del mortiño y finalmente la mora. Las concentraciones de extracto de fruta de 1 y $2 \%$ presentaron un igual poder inhibitorio y a la vez menor a $13 \%$. El análisis de aceptabilidad dio como resultado que la mezcla leche-mortiño tuvo la mayor aceptación, con una calificación de "me gusta moderadamente".

\section{LITERATURA CITADA}

Arreguín-Cano, J., Gerónimo, C., Bermúdez, C., Ostia, M., Ventura, J., Álvarez, C., González, Z. y Gutiérrez-Venegas, G. 2016. Caries dental y microorganismos asociados a la caries en la saliva de los alumnos del primer año de la Facultad de Odontología, UNAM. Revista Odontológica Mexicana 20(2):77-81

Bouarab-Chibane, L., Degraeve, P., Ferhout, H., Bouajila, J., and Oulahal, N. 2019. Plant antimicrobial polyphenols as potential natural food preservatives. Journal of the Science of Food and Agriculture 99:1457-1474.

Cid, M., Martínez, I. y Morales, J. 2008. Ingestión de azúcares en niños menores de 1 año. Revista Médica Electrónica 28(1):113-116.

Cushnie, T. and Lamb, A. 2011. Recent advances in understanding the antibacterial properties of flavonoids. International Journal of Antimicrobial Agents 38:99-107.

Duque de Estrada, J., Pérez, J. y Hidalgo, I. 2006. Caries dental y ecología bucal, aspectos importantes a considerar. Revista Cubana de Estomatología 43(1).

Freire, A., Farfán, A. y Chuquimarca, B. 2016. Elevado consumo de azúcares y caries asociados a cepillado dental en niños de Centros Infantiles del Buen Vivir (CIBVs) de Quito. Revista de la Facultad de Ciencias Médicas (Quito) 41(1):21-30

Gaytán-Andrade, J., Salas, S., López, L., Cobos, P. y Silva, B. 2019. Desarrollo y Evaluación Sensorial De Un Postre de Gelatina Funcional Del Fruto Rojo de Stenocereus queretaroensis (F.A.C. Weber) Buxbaum. Investigación y Desarrollo en Ciencia y Tecnología de Alimentos 4:576-580

GBDS (Global Burden of Disease Study). 2018. Global, regional, and national incidence, prevalence, and years lived with disability for 354 diseases and injuries for 195 countries and territories, 1990-2017: a systematic analysis for the Global Burden of Disease Study 2017. Lancet 392: 1789-8583

Kocevar Glavac, N., and Lunder, M. 2018. Preservative efficacy of selected antimicrobials of natural origin in a cosmetic emulsion. International Journal of Cosmetic Science 40: 276-284.

Lou, Z., Wang, H., Rao, S., Sun, J., Ma, C. and Li, J. 2012. p-Coumaric acid kills bacteria through dual damage mechanisms. Food Control 25: 550-554.

NCCLS (National Committee for Clinical Laboratory Standards). 2015. Performance standards for antimicrobial disk susceptibility test. National Committee for Clinical Laboratory Standards 35:1722.

Orhan, D., Özçelik, B., Özgen, S. and Ergun, F. 2010. Antibacterial, antifungal, and antiviral activities of some flavonoids. Microbiological Research 165: 496-500.

PAE (Programa de Alimentación Escolar). 2017. Ministerio de Inclusión Económica y Social, Quito, Ecuador.

Pérez-Domínguez, J., González-García, A., NieblaFuentes, M. y Ascencio-Montiel, I. 2010. Encuesta de prevalencia de caries dental en niños y adolescentes Revista Médica del Instituto Mexicano del Seguro Social. 48(1): 25-29

Reyes, Y., Cruz, V., Castro, M., Santacruz, S., Villacres, C. y Armas, A. 2019a. Efecto antibacteriano de extractos de Prunus salicifolia (capulí) y Vaccinium floribundum (mortiño) sobre cepas de Streptococcus mutans: Estudio in vitro. KIRU 16(1): 14-18.

Reyes, Y., Santacruz, S., Castro, M., Villacres, C., Chávez, M. y Armas, A. 2019b. Efecto antibacteriano y antioxidante de frutos rojos ecuatorianos sobre streptococcus mutans: estudio in vitro. Odontología Vital 31: 23-30.

Rodríguez, C., Zarate, A. y Sánchez, L. 2017. Actividad antimicrobiana de cuatro variedades de plantas frente 
a patógenos de importancia clínica en Colombia. NOVA 15(27): 119-129

Ruiz, E. y Varela-Moreiras, G. 2017. Adecuación de la ingesta de azúcares totales y añadidos en la dieta española a las recomendaciones: estudio ANIBES. Nutrición Hospitalaria 34: 45-52.
Santacruz, S. and Castro, M. 2018. Viability of free and encapsulated Lactobacillus acidophilus incorporated to cassava starch edible films and its application to Manaba fresh white cheese. LWT Food Science and Technology 93: 570-572.

Vasco, C. 2009. Phenolic Compounds in Ecuadorian Fruits. Ph.D. Thesis. Swedish University of Agricultural Sciences. Uppsala. 